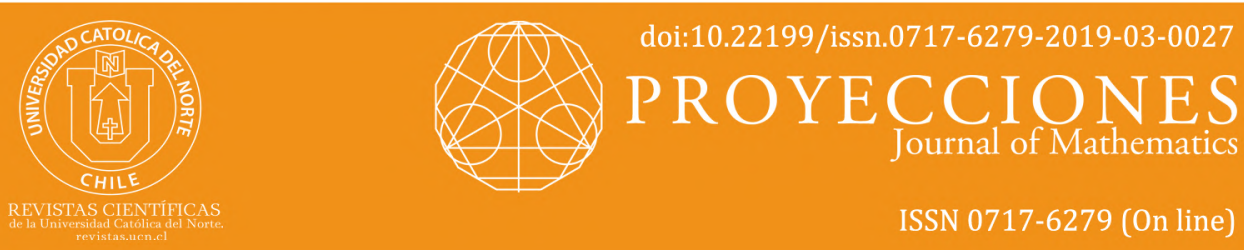

\title{
Odd harmonious labeling of grid graph
}

\section{P. Jeyanthi* (iD orcid.org/0000-0003-4349-164X \\ S. Philo** \\ M. Youssef $^{* * *}$ iD orcid.org/0000-0002-0365-1891}

${ }^{*}$ Govindammal Aditanar College for Women, Dept. of Mathematics, Research Centre, Tiruchendur, TN, India. $\nabla$ jeyajeyanthi@rediffimail.com

${ }^{* *}$ Manonmaniam Sundaranar University. Research Scholar, Reg. No: 12193, Tirunelveli, TN, India.

V lavernejudia@gmail.com

** Imam Mohammad Ibn Saud Islamic University, Dept. of Mathematics and Statistics, College of Science, Riyad, Saudi Arabia. $\nabla$ mzyoussef11566@yahoo.com

Received: February 2018 | Accepted: November 2018

\section{Abstract:}

Agraph $G(p, q)$ is said to be odd harmonious if there exists an injection $f: V(G) \rightarrow\{0,1,2, \cdots, 2 q-1\}$ such that the induced function $f^{*}: E(G) \rightarrow\{1,3, \cdots, 2 q-1\}$ defined by $f^{*}(u v)=f(u)+f(v)$ is a bijection. In this paper we prove that path union of t copies of $P_{m} \times P_{n}$, path union of $t$ different copies of $P_{m_{i}} \times P_{n_{i}}$ where $1 \leq i \leq$ $t$, vertex union of $t$ copies of $P_{m} \times P_{n}$ vertex union of $t$ different copies of $P_{m_{i}} \times P_{n_{i}}$ where $1 \leq i \leq t$, one point union of path of $P_{n}$ (t.n. $\left.P_{m} \times P_{m}\right)$, t super subdivision of grid graph $P_{m} \times P_{n}$ are odd harmonious graphs.

Keywords: Harmonious labeling; Odd harmonious labeling; Grid graph; Path union of graphs; One point union of path of graphs; t-super subdivision of graphs.

MSC (2000): 05C78.

Cite this article as (IEEE citation style):

P. Jeyanthi, S. Philo and M. Youssef, "Odd harmonious labeling of grid graph", Proyecciones (Antofagasta, On line), vol. 38, no. 3, pp. 411-428, Aug. 2019, doi: 10.22199/issn.0717-62792019-03-0027. [Accessed dd-mm-yyyy].

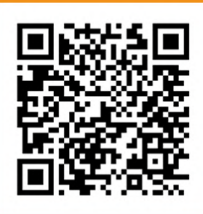

Article copyright: (c) 2019 Pon Jeyanthi, S. Philo and Maged Z. Youssef. This is an open access article distributed under the terms of the Creative Commons Licence, which permits unrestricted use and distribution provided the original author and source are credited. 


\section{Introduction}

Throughout this paper by a graph we mean a finite, simple and undirected one. For standard terminology and notation we follow Harary [3]. A graph $G(V, E)$ with $p$ vertices and $q$ edges is called a $(p, q)$ - graph. The graph labeling is an assignment of integers to the set of vertices or edges or both, subject to certain conditions. An extensive survey of various graph labeling problems is available in [1]. Graham and Sloane [2] introduced harmonious labeling during their study of modular versions of additive bases problems stemming from error correcting codes. A graph $G$ is said to be harmonious if there exists an injection $f: V(G) \rightarrow Z_{q}$ such that the induced function $f^{*}: E(G) \rightarrow Z_{q}$ defined by $f^{*}(u v)=(f(u)+f(v)) \quad(\bmod q)$ is a bijection and $f$ is called harmonious labeling of $G$. The concept of an odd harmonious labeling was due to Liang and Bai [4]. A graph $G$ is said to be odd harmonious if there exists an injection $f: V(G) \rightarrow\{0,1,2, \cdots, 2 q-1\}$ such that the induced function $f^{*}: E(G) \rightarrow\{1,3, \cdots, 2 q-1\}$ defined by $f^{*}(u v)=f(u)+f(v)$ is a bijection. If $f(V(G))=\{0,1,2, \ldots . q\}$ then $f$ is called as strongly odd harmonious labeling and $G$ is called as strongly odd harmonious graph.The odd harmoniousness of graph is useful for the solution of undetermined equations. The following results have been published in $[4]$.

1. If $G$ is an odd harmonious graph, then $G$ is a bipartite graph. Hence any graph that contains an odd cycle is not odd harmonious.

2. If a $(p, q)$-graph $G$ is odd harmonious, then $2 \sqrt{q} \leq p \leq(2 q-1)$.

3. If $G$ is an odd harmonious Eulerian graph with $q$ edges, then $q \equiv$ $0,2(\bmod 4)$.

Jeyanthi et al. [5]-[12] proved that the shadow and splitting of graph $K_{2, n}, C_{n}$ for $n \equiv 0(\bmod 4), H_{n, n}$, any two even cycles sharing a common vertex and a common edge, plus graph $P l_{n}$, open star of plus graph $S\left(t . P l_{n}\right)$, path union of plus graph $P l_{n}$, joining of $C_{m}$ and plus graph $P l_{n}$ with a path, one point union of path of plus graph $P_{n}^{t}\left(t . n . P l_{m}\right)$, super subdivision of any cycle $C_{m}$ with $m \geq 3$,ladder, cycle $C_{n}$ for $n \equiv 0(\bmod 4)$ with $K_{1, m}$, m-shadow and m-splitting of the graphs $P_{n}, H_{n, n}, K_{r, s}, P_{n} \oplus \overline{K_{2}}$ and $S_{p l}\left(C_{n}\right), n \equiv 0(\bmod 4)$ subdivided shell graphs, $S_{m, n}$, spiders, $m$ - 
shadow and $m$-splitting graphs are odd harmonious graphs. Selvaraju et al. [13] proved that $K_{1, n}, S\left(K_{1, n}\right), T_{m, n, t}$ and $S\left(T_{m, n, t}\right)$ are odd harmonious. Vaidya and Shah [14], [15] proved that shadow and splitting graphs of $P_{n}, K_{1, n}, B_{n, n}$ and super subdivision of $H_{n, n}$ are odd harmonious.

We use the following definitions in the subsequent section.

Definition 1. The Cartesian product of graphs $G$ and $H$ denoted as $G \times H$, is the graph with vertex set $V(G) \times V(H)=\{(u, v) / u \in V(G)$ and $v \in$ $V(H)\}$ and $(u, v)$ is adjacent to $(u, v)$ if and only if either $u=u$, and edge $\left(v, v^{\prime}\right) \in E(H)$ or $v=v$, and edge $\left(u, u^{\prime}\right) \in E(G)$. The Cartesian product of two paths $P_{m}$ and $P_{n}$ denoted by $P_{m} \times P_{n}$ is known as a grid graph on $m n$ vertices and $2 m n-(m+n)$ edges.

Definition 2. Let $G$ be a graph and $G_{1}, G_{2}, \cdots, G_{n}, n \geq 2$ be $n$ copies of graph $G$.Then the graph obtained by adding an edge from $G_{i}$ to $G_{i+1}$ $(i=1,2, \cdots, n-1)$ is called path union of graph $G$.

Definition 3. A graph $G$ is obtained by replacing each edge of $K_{1, t}$ by a path $P_{n}$ of length $n$ on $n+1$ vertices is called one point union for $t$ copies of path $P_{n}$, denoted by $P_{n}^{t}$.

Definition 4. A graph $G$ is obtained by replacing each vertices of $P_{n}^{t}$ except the central vertex by the graphs $G_{1}, G_{2}, \cdots, G_{n}$ is known as one point union for path of graphs, denoted by $P_{n}^{t}\left(G_{1}, G_{2}, \cdots, G_{n}\right)$ where $P_{n}^{t}$ is the one point union of $t$ copies of path $P_{n}$. If we replace each vertices of $P_{n}^{t}$ except the central vertex by the graph $H$, that is $G_{1}=G_{2}=G_{3}=\cdots G_{n}=H$, such one point union of path graph, denoted by $P_{n}^{t}($ t.n.H).

Definition 5. Let $G=(V, E)$ be a graph with $p$ vertices and $q$ edges. A graph $H$ is said to be a t-super subdivision of $G$ if $H$ is obtained from $G$ by replacing every edge $e$ of $G$ by a complete bipartite graph $K_{2, t}$ for some $t \in N$.

\section{Main Results}

Theorem 2.1. Path union of $t$ copies of $P_{m} \times P_{n}$ is odd harmonious, where $m, n, t \geq 2$. 
Proof. $\quad$ Let $G$ be a path union of $t$ copies of $P_{m} \times P_{n}, \forall m, n, t \geq 2$.

Let $x_{k, i, 1}, x_{k, i, 2}, \cdots, x_{k, i, n}$ be the vertices of the $k^{t h}$ copy of $i^{t h}$ row vertices in $G$, where $1 \leq k \leq t, 1 \leq i \leq m$. Now, we join these consecutive copies of the grid graph $P_{m} \times P_{n}$ by an edge. Join $x_{k, m, n}$ with $x_{k+1,1,1}$, $\forall 1 \leq k \leq t-1$ by an edge to form a path union of $t$ copies of the grid grpah $P_{m} \times P_{n}$.

Then $|V(G)|=t m n$ and $|E(G)|=t[2 m n-(m+n)+1]-1$.

We define a labeling $f: V(G) \rightarrow\{0,1,2, \cdots, 2\{t[2 m n-(m+n)+1]-1\}-1\}$ as follows:

$$
f\left(x_{k, i, j)}=(i-1)(2 n-1)+j-1+(k-1)[m(2 n-1)-n+1], 1 \leq i \leq m,\right.
$$
$1 \leq j \leq n, 1 \leq k \leq t$.

The induced edge labels are

$$
f^{*}\left(x_{k, i, j} x_{k, i, j+1}\right)=2(i-1)(2 n-1)+2 j-1+2(k-1)[m(2 n-1)-n+1],
$$
$1 \leq i \leq m, 1 \leq j \leq n-1,1 \leq k \leq t$

$$
f^{*}\left(x_{k, i, j} x_{k, i+1, j}\right)=2 i(2 n-1)+2(j-1)-2 n+1+2(k-1)[m(2 n-1)-n+1],
$$
$1 \leq i \leq m-1,1 \leq j \leq n, 1 \leq k \leq t$

$$
f^{*}\left(x_{k, m, n} x_{k+1,1,1}\right)=2 k[m(2 n-1)-n+1]-1,1 \leq k \leq t-1 .
$$

In view of the above defined labeling pattern, path union of $t$ copies of $P_{m} \times P_{n}$ is odd harmonious, where $m, n, t \geq 2$.

An odd harmonious labeling of path union of 3 copies of $P_{3} \times P_{4}$ is shown in Figure 1. 


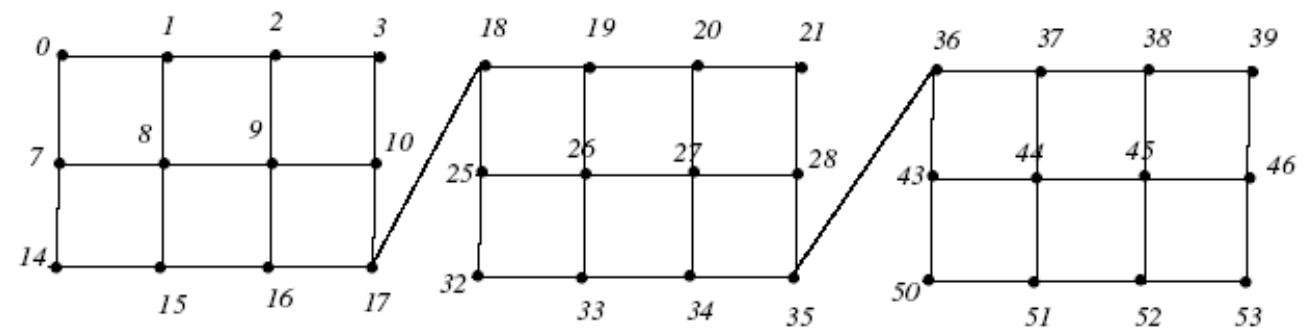

Figure 1: An odd harmonious labeling of path union of 3 copies of $P_{3} \times P_{4}$.

Theorem 2.2. Path union of $t$ different copies of $P_{m_{i}} \times P_{n_{i}}, 1 \leq i \leq t$ is odd harmonious.

Proof. Let $G$ be a path union of $t$ different copies of the grid graph $P_{m_{i}} \times P_{n_{i}}, 1 \leq i \leq t$. Let $x_{k, i, 1}, x_{k, i, 2}, \cdots, x_{k, i, n_{k}}$ be the vertices of the $k^{t h}$ copy of the $i^{\text {th }}$ row vertices in $G$, where $1 \leq i \leq m_{k}, 1 \leq k \leq t$. Now, we join these consecutive copies of the grid graph $P_{m_{i}} \times P_{n_{i}}, 1 \leq i \leq t$ by an edge. Join $x_{k, m_{k}, n_{k}}$ with $x_{k+1,1,1}, \forall 1 \leq k \leq t-1$ by an edge to form a path union of $t$ copies of the grid graph $P_{m_{i}} \times P_{n_{i}}, 1 \leq i \leq t$.

Then $|V(G)|=n_{1} m_{1}+n_{2} m_{2}+\cdots+n_{t} m_{t}$ and $|E(G)|=2\left(m_{1} n_{1}+\right.$ $\left.m_{2} n_{2}+\cdots+m_{t} n_{t}\right)-\left(m_{1}+m_{2}+\cdots+m_{t}\right)-\left(n_{1}+n_{2}+\cdots+n_{t}\right)+(t-1)$.

We define a labeling $f: V(G) \rightarrow\{0,1,2, \cdots, 2|E(G)|-1\}$ as follows:

$$
\begin{aligned}
& \quad f\left(x_{k, i, j}\right)=2\left[m_{1} n_{1}+m_{2} n_{2}+\cdots+m_{k-1} n_{k-1}\right]-\left[m_{1}+m_{2}+\cdots+m_{k-1}\right]- \\
& {\left[n_{1}+n_{2}+\cdots+n_{k-1}\right]+2 n_{k}(i-1)+j-i+(k-1), 1 \leq k \leq t, 1 \leq i \leq m_{k}} \\
& 1 \leq j \leq n_{k} .
\end{aligned}
$$

The induced edge labels are

$$
\begin{aligned}
& f^{*}\left(x_{k, i, j} x_{k, i, j+1}\right)=4\left[m_{1} n_{1}+m_{2} n_{2}+\cdots+m_{k-1} n_{k-1}\right]-2\left[m_{1}+m_{2}+\cdots+\right. \\
& \left.m_{k-1}\right]-2\left[n_{1}+n_{2}+\cdots+n_{k-1}\right]+4 n_{k}(i-1)+2 j-2 i+2 k-1,1 \leq k \leq t \\
& 1 \leq i \leq m_{k}, 1 \leq j \leq n_{k}-1
\end{aligned}
$$


$f^{*}\left(x_{k, i, j} x_{k, i+1, j}\right)=4\left[m_{1} n_{1}+m_{2} n_{2}+\cdots+m_{k-1} n_{k-1}\right]-2\left[m_{1}+m_{2}+\cdots+\right.$ $\left.m_{k-1}\right]-2\left[n_{1}+n_{2}+\cdots+n_{k-1}\right]+2 n_{k}(2 i-1)+2 j-2 i+2 k-3,1 \leq k \leq t$, $1 \leq i \leq m_{k}-1,1 \leq j \leq n_{k}$

$$
f^{*}\left(x_{k, m_{k}, n_{k}} x_{k+1,1,1}\right)=4\left[m_{1} n_{1}+m_{2} n_{2}+\cdots+m_{k-1} n_{k-1}\right]-2\left[m_{1}+m_{2}+\right.
$$
$\left.\cdots+m_{k-1}\right]-2\left[n_{1}+n_{2}+\cdots+n_{k-1}\right]+4 m_{k} n_{k}-2 m_{k}-2 n_{k}+2 k-1,1 \leq k \leq t-1$.

In view of the above defined labeling pattern, path union of $t$ different copies of $P_{m_{i}} \times P_{n_{i}}, 1 \leq i \leq t$ is odd harmonious .

An odd harmonious labeling of path union of 2 copies $P_{5} \times P_{4}$ and $P_{6} \times P_{8}$ is shown in Figure 2.
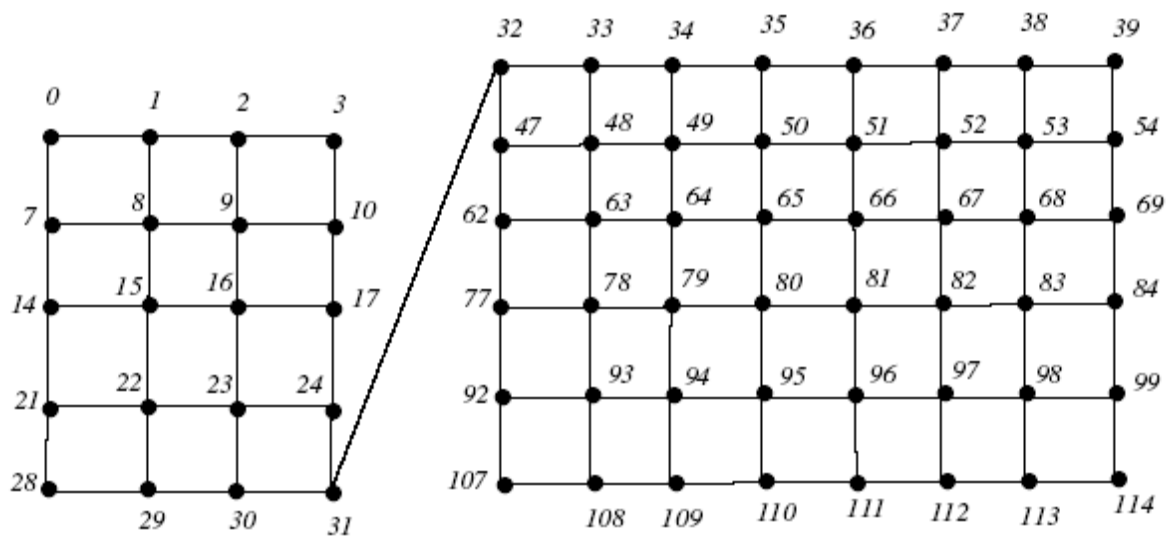

Figure 2:An odd harmonious labeling of path union of 2 copies $P_{5} \times P_{4}$ and $P_{6} \times P_{8}$.

Theorem 2.3. The vertex union of $t$ copies of $P_{m} \times P_{n}$ is odd harmonious, where $m, n \geq 2$.

Proof. Let $G$ be a vertex union of $t$ copies of the grid graph $P_{m} \times P_{n}$, $\forall m, n, t \geq 2$. Let $x_{k, i, 1}, x_{k, i, 2}, \cdots, x_{k, i, n}$ be the vertices of the $k^{t h}$ copy of the $i^{\text {th }}$ row vertices in $G$, where $1 \leq k \leq t, 1 \leq i \leq m$. Now, we join these consecutive copies of the grid graph $P_{m} \times P_{n}$ by a vertex. Identifying the vertices $x_{k, m, n}$ with $x_{k+1,1,1}, \forall 1 \leq k \leq t-1$ to form a vertex union of $t$ copies of the grid graph $P_{m} \times P_{n}$. 
Case (i): When $m=n$

Then $|V(G)|=t n^{2}-(t-1)$ and $|E(G)|=2 n(n-1) t$.

We define a labeling $f: V(G) \rightarrow\{0,1,2, \cdots, 2[2 n(n-1) t]-1\}$ as follows:

$f\left(x_{k, i, j)}=(i-1)(2 n-1)+j-1+(k-1)[2 n(n-1)], 1 \leq i, j \leq n\right.$, $1 \leq k \leq t$

The induced edge labels are

$f^{*}\left(x_{k, i, j} x_{k, i, j+1}\right)=2(i-1)(2 n-1)+2 j-1+2(k-1)[2 n(n-1)], 1 \leq i \leq n$, $1 \leq j \leq n-1,1 \leq k \leq t$

$f^{*}\left(x_{k, i, j} x_{k, i+1, j}\right)=2 i(2 n-1)+2(j-1)-2 n+1+2(k-1)[2 n(n-1)]$, $1 \leq i \leq n-1,1 \leq j \leq n, 1 \leq k \leq t$.

Case(ii): When $m \neq n$

Then $|V(G)|=$ tmn $-(t-1)$ and $|E(G)|=[(m-1)(2 n-1)+n-1]$.

We define a labeling $f: V(G) \rightarrow\{0,1,2, \cdots, 2[(m-1)(2 n-1)+n-1] t-1\}$ as follows:

$$
f\left(x_{k, i, j)}=(i-1)(2 n-1)+j-1+(k-1)[(m-1)(2 n-1)+n-1],\right.
$$

$1 \leq i \leq m, 1 \leq j \leq n, 1 \leq k \leq t$.

The induced edge labels are

$$
f^{*}\left(x_{k, i, j} x_{k, i, j+1}\right)=2(i-1)(2 n-1)+2 j-1+2(k-1)[(m-1)(2 n-1)+n-1],
$$
$1 \leq i \leq m, 1 \leq j \leq n-1,1 \leq k \leq t$

$$
f^{*}\left(x_{k, i, j} x_{k, i+1, j}\right)=2 i(2 n-1)+2(j-1)-2 n+1+2(k-1)[(m-1)(2 n-
$$
1) $+n-1], 1 \leq i \leq m-1,1 \leq j \leq n, 1 \leq k \leq t$.

In view of above defined labeling pattern,the vertex union of $t$ copies of $P_{m} \times P_{n}$ is odd harmonious, where $m, n \geq 2$.

An odd harmonious labeling of vertex union of 3 copies of $P_{3} \times P_{3}$ $(m=n)$, is shown in Figure 3. 


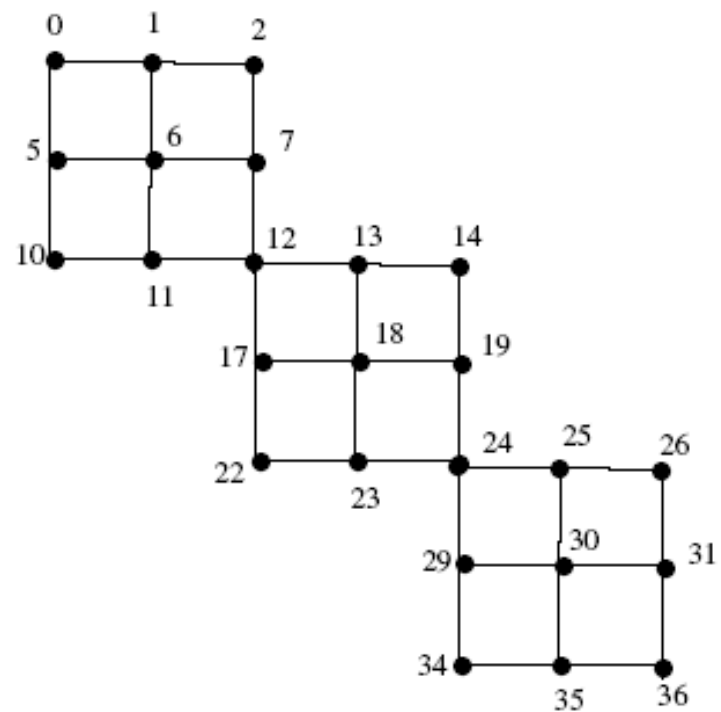

Figure 3: An odd harmonious labeling of vertex union of 3 copies of $P_{3} \times P_{3}$.

An odd harmonious labeling of vertex union of 2 copies of $P_{3} \times P_{4}$ $(m \neq n)$ is shown in Figure 4 .

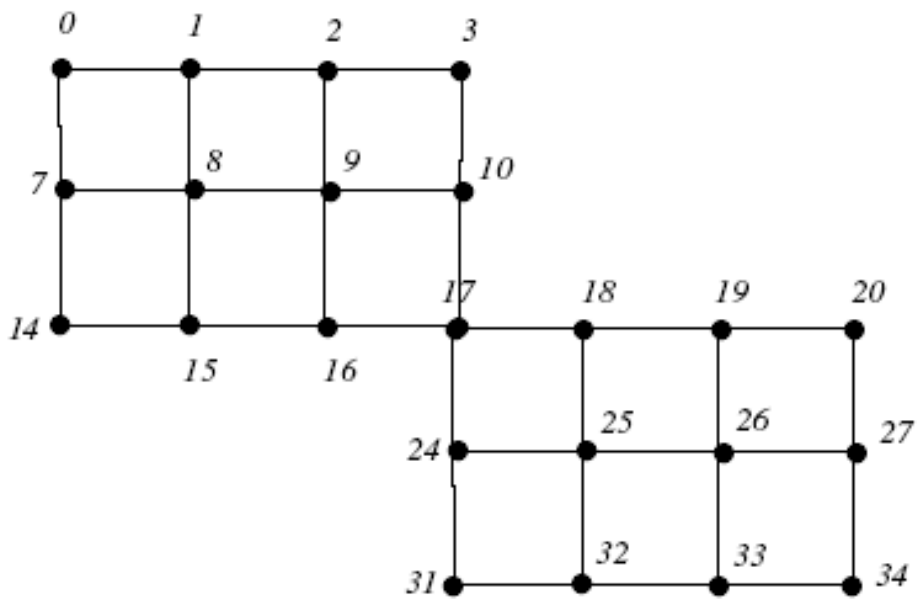

Figure 4: An odd harmonious labeling of vertex union of 2 copies of $P_{3} \times P_{4}$. 
Theorem 2.4. The vertex union of $t$ different copies of the grid graph $P_{m_{i}} \times P_{n_{i}}, 1 \leq i \leq t$ is odd harmonious.

Proof. Let $G$ be a vertex union of $t$ different copies of the grid graph $P_{m_{i}} \times P_{n_{i}}, 1 \leq i \leq t$. Let $x_{k, i, 1}, x_{k, i, 2}, \cdots, x_{k, i, n_{k}}$ be the vertices of the $k^{t h}$ copy of the $i^{t h}$ row vertices in $G$, where $1 \leq i \leq m_{k}, 1 \leq k \leq t$. Now, we join these consecutive copies of the grid graph $P_{m_{i}} \times P_{n_{i}}, 1 \leq i \leq t$ by a vertex. Identifying the vertices $x_{k, m_{k}, n_{k}}$ with $x_{k+1,1,1}, \forall 1 \leq k \leq t-1$ to form a vertex union of $t$ copies of the grid graph $P_{m_{i}} \times P_{n_{i}}, 1 \leq i \leq t$.

Then $|V(G)|=\left(n_{1} m_{1}+n_{2} m_{2}+\cdots+n_{t} m_{t}\right)-(t-1)$ and

$|E(G)|=2\left(m_{1} n_{1}+m_{2} n_{2}+\cdots+m_{t} n_{t}\right)-\left(m_{1}+m_{2}+\cdots+m_{t}\right)-\left(n_{1}+\right.$ $\left.n_{2}+\cdots+n_{t}\right)$.

We define a labeling $f: V(G) \rightarrow\{0,1,2, \cdots, 2|E(G)|-1\}$ as follows:

$$
\begin{gathered}
f\left(x_{k, 1, j)}=2\left[m_{1} n_{1}+m_{2} n_{2}+\cdots+m_{k-1} n_{k-1}\right]-\left[m_{1}+\cdots+m_{k-1}\right]-\left[n_{1}+\right.\right. \\
\left.\cdots+n_{k-1}\right]+(j-1), 1 \leq j \leq n_{k}, 1 \leq k \leq t \\
f\left(x_{k, i, j)}=\left(2 n_{k}-1\right)(i-1)+j-1+2\left[m_{1} n_{1}+m_{2} n_{2}+\cdots+m_{k-1} n_{k-1}\right]-\right. \\
{\left[m_{1}+\cdots+m_{k-1}\right]-\left[n_{1}+\cdots+n_{k-1}\right], 1 \leq j \leq n_{k}, 2 \leq i \leq m_{k}, 1 \leq k \leq t}
\end{gathered}
$$

The induced edge labels are

$$
\begin{gathered}
f^{*}\left(x_{k, 1, j} x_{k, 1, j+1}\right)=4\left[m_{1} n_{1}+\cdots+m_{k-1} n_{k-1}\right]-2\left[m_{1}+\cdots+m_{k-1}\right]- \\
2\left[n_{1}+\cdots+n_{k-1}\right]+2 j-1,1 \leq j \leq n_{k}, 1 \leq k \leq t \\
f^{*}\left(x_{k, 1, j} x_{k, 2, j}\right)=\left(2 n_{k}-1\right)+2(j-1)+4\left[m_{1} n_{1}+\cdots+m_{k-1} n_{k-1}\right]- \\
2\left[m_{1}+\cdots+m_{k-1}\right]-2\left[n_{1}+\cdots+n_{k-1}\right], 1 \leq j \leq n_{k}, 1 \leq k \leq t ; \\
f^{*}\left(x_{k, i, j} x_{k, i, j+1}\right)=2\left(2 n_{k}-1\right)(i-1)+2 j-1+4\left[m_{1} n_{1}+\cdots+m_{k-1} n_{k-1}\right]- \\
2\left[m_{1}+\cdots+m_{k-1}\right]-2\left[n_{1}+\cdots+n_{k-1}\right], 2 \leq i \leq m_{k}, 1 \leq j \leq n_{k}-1,1 \leq k \leq t ; \\
f^{*}\left(x_{k, i, j} x_{k, i+1, j}\right)=\left(2 n_{k}-1\right)(2 i-1)+2(j-1)+4\left[m_{1} n_{1}+\cdots+m_{k-1} n_{k-1}\right]-
\end{gathered}
$$


$2\left[m_{1}+\cdots+m_{k-1}\right]-2\left[n_{1}+\cdots+n_{k-1}\right], 2 \leq i \leq m_{k}-1,1 \leq j \leq n_{k}, 1 \leq k \leq t$.

In the view of above defined labeling pattern, vertex union of $t$ different copies of the grid graph $P_{m_{i}} \times P_{n_{i}}, 1 \leq i \leq t$ is odd harmonious.

An odd harmonious labeling of vertex union of 3 different copies of $P_{3} \times P_{3}, P_{3} \times P_{4}, P_{4} \times P_{5}$ is shown in Figure 5.

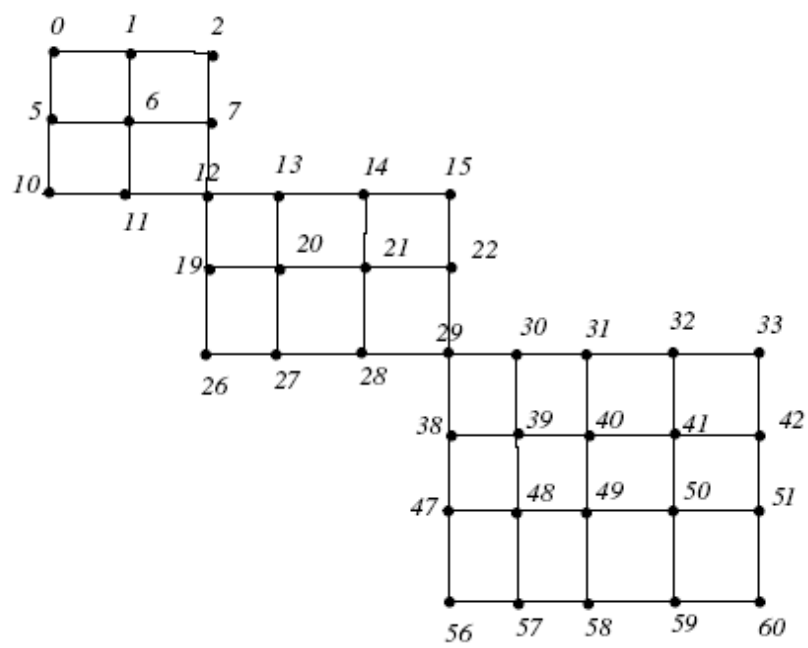

Figure 5:An odd harmonious labeling of vertex union of 3 different copies $P_{3} \times P_{3}, P_{3} \times P_{4}, P_{4} \times P_{5}$.

Theorem 2.5. The $t$-super subdivision of grid graph $P_{m} \times P_{n}$ is odd harmonious, where $m, n \geq 2$.

Proof. Let $x_{i, j}(1 \leq i \leq n, 1 \leq j \leq m)$ be the vertices of the grid graph $P_{m} \times P_{n}$. We know that the number of vertices in $P_{m} \times P_{n}$ is $p=m n$ and the number of edges $q=2 m n-(m+n)$. Let $G$ be a graph obtained by $t$-super subdivision of $P_{m} \times P_{n}$. Then we see that the number of vertices in $G$ is $P=|V(G)|=p+t q$ and the number of edges in $G$ is $Q=|E(G)|=2 t q$.

Let $u_{i, j, k}(1 \leq i \leq n-1,1 \leq j \leq m, 1 \leq k \leq t)$ be vertices for the vertical edges in $G$ and $v_{i, j, k}(1 \leq i \leq n, 1 \leq j \leq m-1,1 \leq k \leq t)$ be 
vertices for horizontal edges in $G$.

We define the labeling $f: V(G) \rightarrow\{0,1,2, \cdots, 4 t q-1\}$ as follows:

$$
\begin{aligned}
& f\left(x_{i, j}\right)=2 t(i-1)(2 m-1)+2 t(j-1), \text { if } i \text { is odd, } 1 \leq j \leq m ; \\
& f\left(x_{i, j)}=2 t(i-1)(2 m-1)+2 t(m-j), \text { if } i \text { is even, } 1 \leq j \leq m ;\right. \\
& f\left(v_{1, j, k)}=2 t(j-1)+2 k-1,1 \leq j \leq m-1,1 \leq k \leq t ;\right. \\
& f\left(v_{i, j, k)}=2 t(i-2)(2 m-1)+2 t(m-1)+2 t-1+2 k+4 t(j-1),\right. \\
& 1 \leq j \leq m-1, i=3,5, \cdots, 1 \leq k \leq t
\end{aligned}
$$

$$
f\left(v_{i, j, k)}=2 t(i-2)(2 m-1)+2 t(m-1)+2 t-1+2 k+4 t(m-j-1),\right.
$$
$1 \leq j \leq m-1, i=2,4, \cdots, 1 \leq k \leq t$

$$
f\left(u_{i, j, k)}=2 t(i-1)(2 m-1)+2 t(m-1)+2 k-1+4 t(m-j), 1 \leq j \leq m,\right.
$$
$i=3,5, \cdots, 1 \leq k \leq t$

$$
f\left(u_{i, j, k)}=2 t(i-1)(2 m-1)+2 t(m-1)+2 k-1+4 t(j-1), 1 \leq j \leq m,\right.
$$
$i=2,4, \cdots, 1 \leq k \leq t$.

The induced edge labels are

$$
\begin{array}{r}
f^{*}\left(x_{1, j} v_{1, s, k}\right)=2 t(j-1)+2 t(s-1)+2 k-1,1 \leq j \leq m, 1 \leq s \leq m-1 ; \\
f^{*}\left(x_{1, j} u_{1, s, k}\right)=2 t(j-1)+2 t(m-1)+2 k-1+4 t(m-s), 1 \leq j, s \leq m ; \\
f^{*}\left(x_{i, j} u_{l, s, k}\right)=2 t(i-1)(2 m-1)+2 t(m-j)+2 t(l-1)(2 m-1)+2 t(m- \\
1)+2 k-1+4 t(m-s), i=2,4, \cdots, 1 \leq j, s \leq m, l=1,3,5, \cdots, 1 \leq k \leq t ;
\end{array}
$$

$$
f^{*}\left(x_{i, j} u_{l, s, k}\right)=2 t(i-1)(2 m-1)+2 t(m-j)+2 t(l-1)(2 m-1)+2 t(m-
$$

$1)+2 k-1+4 t(s-1), i=2,4, \cdots, 1 \leq j, s \leq m, l=2,4,6, \cdots, 1 \leq k \leq t$;

$$
f^{*}\left(x_{i, j} u_{l, s, k}\right)=2 t(i-1)(2 m-1)+2 t(j-1)+2 t(l-1)(2 m-1)+2 t(m-
$$

1) $+2 k-1+4 t(m-s), i=1,3, \cdots, 1 \leq j, s \leq m, l=1,3,5, \cdots, 1 \leq k \leq t$;

$f^{*}\left(x_{i, j} u_{l, s, k}\right)=2 t(i-1)(2 m-1)+2 t(j-1)+2 t(l-1)(2 m-1)+2 t(m-$ 1) $+2 k-1+4 t(s-1), i=1,3, \cdots, 1 \leq j, s \leq m, l=2,4, \cdots, 1 \leq k \leq t$; 
$f^{*}\left(x_{i, j} v_{l, s, k}\right)=2 t(i-1)(2 m-1)+2 t(j-1)+2 t(l-2)(2 m-1)+2 t(m-$ 1) $+2 t-1+2 k+4 t(s-1), i=1,3, \cdots, 1 \leq j \leq m, 1 \leq s \leq m-1$, $l=3,5, \cdots, 1 \leq k \leq t$

$f^{*}\left(x_{i, j} v_{l, s, k}\right)=2 t(i-1)(2 m-1)+2 t(j-1)+2 t(l-2)(2 m-1)+2 t(m-$ 1) $+2 t-1+2 k+4 t(m-s-1), i=3,5, \cdots, 1 \leq j \leq m, 1 \leq s \leq m-1$, $l=2,4, \cdots, 1 \leq k \leq t$

$$
f^{*}\left(x_{i, j} v_{l, s, k}\right)=2 t(i-1)(2 m-1)+2 t(m-j)+2 t(l-2)(2 m-1)+2 t(m-
$$
1) $+2 t-1+2 k+4 t(s-1), i=2,4, \cdots, 1 \leq j \leq m, 1 \leq s \leq m-1$, $l=3,5, \cdots, 1 \leq k \leq t$

$$
f^{*}\left(x_{i, j} v_{l, s, k}\right)=2 t(i-1)(2 m-1)+2 t(m-j)+2 t(l-2)(2 m-1)+2 t(m-
$$
1) $+2 t-1+2 k+4 t(m-s-1), i=2,4, \cdots, 1 \leq j \leq m, 1 \leq s \leq m-1$, $l=2,4, \cdots, 1 \leq k \leq t$.

In the view of above defined labeling pattern, $t$-super subdivision of $P_{m} \times P_{n}, m, n \geq 2$ is odd harmonious.

An odd harmonious labeling of 2-super subdivision of $P_{3} \times P_{3}$ is shown in Figure 6. 


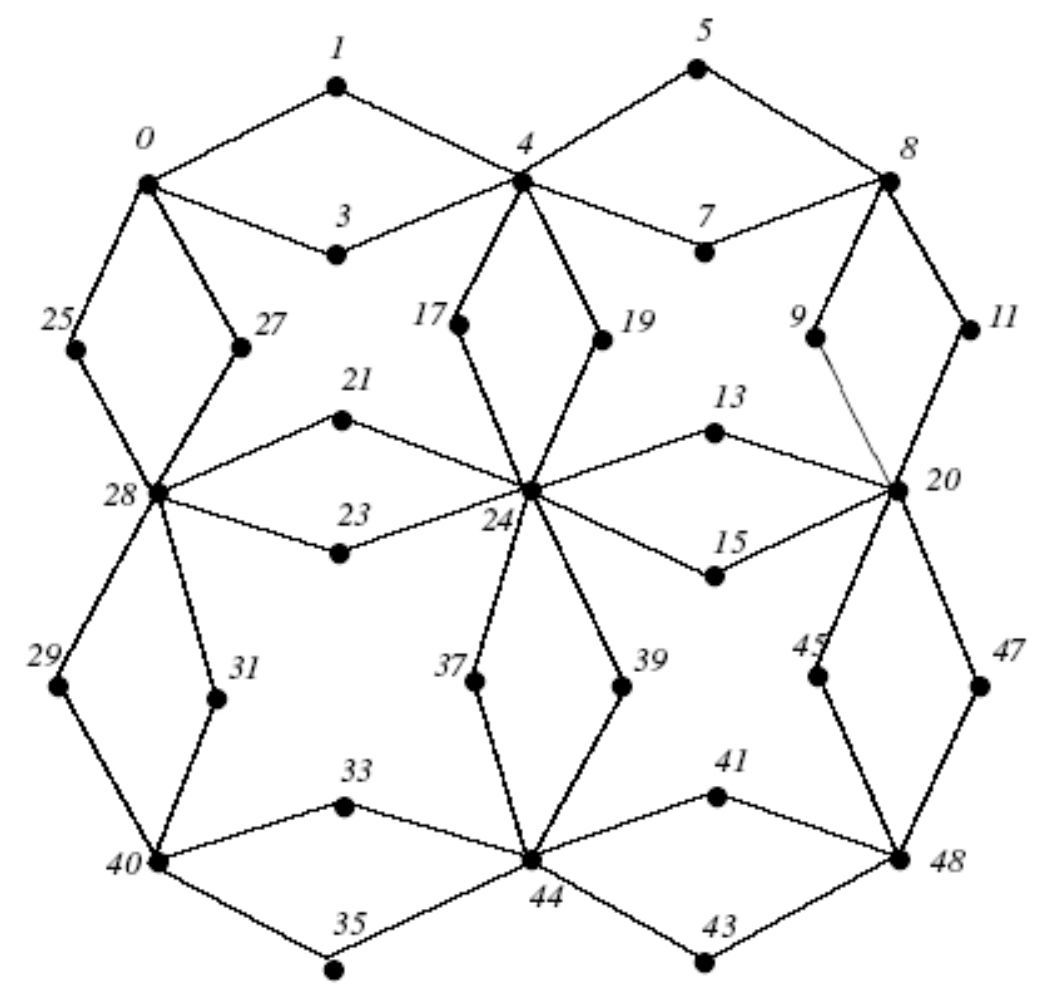

Figure 6:An odd harmonious labeling of 2-super subdivision of $P_{3} \times P_{3}$.

Theorem 2.6. One point union of path of graph $P_{n}^{t}\left(\right.$ t.n. $\left.P_{m} \times P_{m}\right), n \geq 1$, $m \geq 2$ is odd harmonious if $t$ is odd.

Proof. Let $G=P_{n}^{t}\left(\right.$ t.n. $\left.P_{m} \times P_{m}\right)$ be a graph obtained by replacing each vertices of $P_{n}^{t}$ except the central vertex by the graph $P_{m} \times P_{m}$. That means $G$ is the graph obtained by replacing each vertices of $K_{1, t}$ except the apex vertex by the path union of $n$ copies of the graph $P_{m} \times P_{m}$. Let $u_{0}$ be the central vertex for the graph $G$ with $t$ branches. Let $x_{s, k, i, j}$ $(\forall 1 \leq k \leq n, 1 \leq i, j \leq m)$ be the vertices of $k^{\text {th }}$ copy of path union of $n$ copies of $P_{m} \times P_{m}$ lies in the $s^{t h}$ branch of the graph $G, \forall s=1,2, \cdots, t$. 
Join the vertices of $u_{s, 1,1,1}$ with $u_{0}$ by an edge to form the one point union for path of grid graph $G$. Also join the vertices $u_{s, k, m, m}$ to $u_{s, k+1,1,1}$ for $k=1,2, \cdots, n-1, s=1,2, \cdots, t$ by an edge. This graph $G$ with $|V(G)|=t n m^{2}+1$ and $|E(G)|=\operatorname{tn}[2 m(m-1)+1]$.

We define the labeling $f: V(G) \rightarrow\{0,1,2, \cdots, 2 \operatorname{tn}[2 m(m-1)+1]-1\}$ as follows:

$f\left(u_{0}\right)=0$;

$f\left(x_{s, k, i, j}\right)=2+4(t-s)+t(j-1)+t(2 m-1)(i-1)+2 t m(m-1)+$ $t(k-2)[2 m(m-1)+1]$, if both $i$ and $j$ are odd, $k=2,4, \cdots, 1 \leq s \leq t$;

$$
f\left(x_{s, k, i, j}\right)=2 s-1+t(j-2)+t(2 m-1)(i-1)+t[2 m(m-1)+2]+
$$
$t(k-2)[2 m(m-1)+1]$, if $i$ is odd and $j$ is even, $k=2,4, \cdots, 1 \leq s \leq t$;

$$
f\left(x_{s, k, i, j}\right)=2 m t+1+t(j-1)+t(2 m-1)(i-2)+2(s-1)+2 t m(m-
$$
$1)+t(k-2)[2 m(m-1)+1]$, if $i$ is even and $j$ is odd, $k=2,4, \cdots, 1 \leq s \leq t$;

$f\left(x_{s, k, i, j}\right)=2[1+t(m-1)]+t(j-2)+4(t-s)+t(2 m-1)(i-2)+t[2 m(m-$ $1)+2]+t(k-2)[2 m(m-1)+1]$, if both $i$ and $j$ are even, $k=2,4, \cdots$, $1 \leq s \leq t$

$$
f\left(x_{s, k, i, j}\right)=2 s-1+t(j-1)+t(2 m-1)(i-1)+t(k-1)[2 m(m-1)+1],
$$
if both $i$ and $j$ are odd, $k=1,3, \cdots, 1 \leq s \leq t$;

$$
f\left(x_{s, k, i, j}\right)=2+4(t-s)+t(j-2)+t(2 m-1)(i-1)+t(k-1)[2 m(m-1)+1],
$$
if $i$ is odd and $j$ is even, $k=1,3, \cdots, 1 \leq s \leq t$;

$f\left(x_{s, k, i, j}\right)=2[1+t(m-1)]+t(j-1)+4(t-s)+t(2 m-1)(i-2)+$ $t(k-1)[2 m(m-1)+1]$, if $i$ is even and $j$ is odd, $k=1,3, \cdots, 1 \leq s \leq t$;

$$
f\left(x_{s, k, i, j}\right)=2 m t+1+t(j-2)+t(2 m-1)(i-2)+2(s-1)+t(k-
$$
1) $[2 m(m-1)+1]$, if both $i$ and $j$ are even, $k=1,3, \cdots, 1 \leq s \leq t$.

The induced edge labels are

$f^{*}\left(x_{s, k, i, j} x_{s, k, i, j+1}\right)=1-2 s+4 t+2 t(j-1)+2 t(2 m-1)(i-1)+2 t(k-$ 1) $[2 m(m-1)+1]$, if $k$ and $i$ are odd, $1 \leq j \leq m-1,1 \leq s \leq t$; 
$f^{*}\left(x_{s, k, i, j} x_{s, k, i, j+1}\right)=1-2 s+2 t(2 m+1)+2 t(j-1)+2 t(2 m-1)(i-$ $2)+2 t(k-1)[2 m(m-1)+1]$, if $k$ is odd and $i$ is even, $1 \leq j \leq m-1$, $1 \leq s \leq t$

$$
f^{*}\left(x_{s, k, i, j} x_{s, k, i, j+1}\right)=1-2 s+6 t+2 t(j-1)+2 t(2 m-1)(i-1)+4 t m(m-
$$
$1)+2 t(k-2)[2 m(m-1)+1]$, if $k$ is even and $i$ is odd, $1 \leq j \leq m-1$, $1 \leq s \leq t$

$$
f^{*}\left(x_{s, k, i, j} x_{s, k, i, j+1}\right)=1-2 s+4 t+4 t m^{2}+2 t(j-1)+2 t(2 m-1)(i-
$$
$2)+2 t(k-2)[2 m(m-1)+1]$, if both $k$ and $i$ are even, $1 \leq j \leq m-1$, $1 \leq s \leq t$

$$
f^{*}\left(x_{s, k, i, j} x_{s, k, i+1, j}\right)=1-2 s+2 t(m+1)+2 t(j-1)+2 t(2 m-1)(i-
$$
$1)+2 t(k-1)[2 m(m-1)+1]$, if $k$ and $j$ are odd, $1 \leq i \leq m-1,1 \leq s \leq t$;

$$
f^{*}\left(x_{s, k, i, j} x_{s, k, i+1, j}\right)=1-2 s+2 t(m+2)+2 t(j-2)+2 t(2 m-1)(i-
$$
$1)+2 t(k-1)[2 m(m-1)+1]$, if $k$ is odd and $j$ is even, $1 \leq i \leq m-1$, $1 \leq s \leq t$

$$
f^{*}\left(x_{s, k, i, j} x_{s, k, i+1, j}\right)=1-2 s+2 t(m+2)+2 t(j-1)+2 t(2 m-1)(i-1)+
$$
$4 t m(m-1)+2 t(k-2)[2 m(m-1)+1]$, if $k$ is even and $j$ is odd, $1 \leq i \leq m-1$, $1 \leq s \leq t$

$f^{*}\left(x_{s, k, i, j} x_{s, k, i+1, j}\right)=1-2 s+6 t+2 t m+2 t(j-2)+4 t m(m-1)+2 t(2 m-$ 1) $(i-1)+2 t(k-2)[2 m(m-1)+1]$, if $k$ and $j$ are even, $1 \leq i \leq m-1$, $1 \leq s \leq t$

$f^{*}\left(x_{s, k, m, m} x_{s, k+1,1,1}\right)=1-2 s+4 t+4 t m(m-1)+2 t(k-1)[2 m(m-1)+1]$, if $m$ is odd, $1 \leq k \leq m-1,1 \leq s \leq t$;

$f^{*}\left(x_{s, k, m, m} x_{s, k+1,1,1}\right)=1-2 s+2 t+t m(2 m+1)+t(m-2)(2 m-1)+$ $2 t(k-1)[2 m(m-1)+1]$, if $m$ is even, $1 \leq k \leq m-1,1 \leq s \leq t$.

In the view of above defined labeling pattern, one point union of path of grid graph $P_{n}^{t}\left(\right.$ t.n. $\left.P_{m} \times P_{m}\right), n \geq 1, m \geq 2$ is odd harmonious if $t$ is odd.

An odd harmonious labeling of one point union of path of grid graph $P_{2}^{3}\left(3.2 . P_{4} \times P_{4}\right)$ is shown in Figure 7 . 


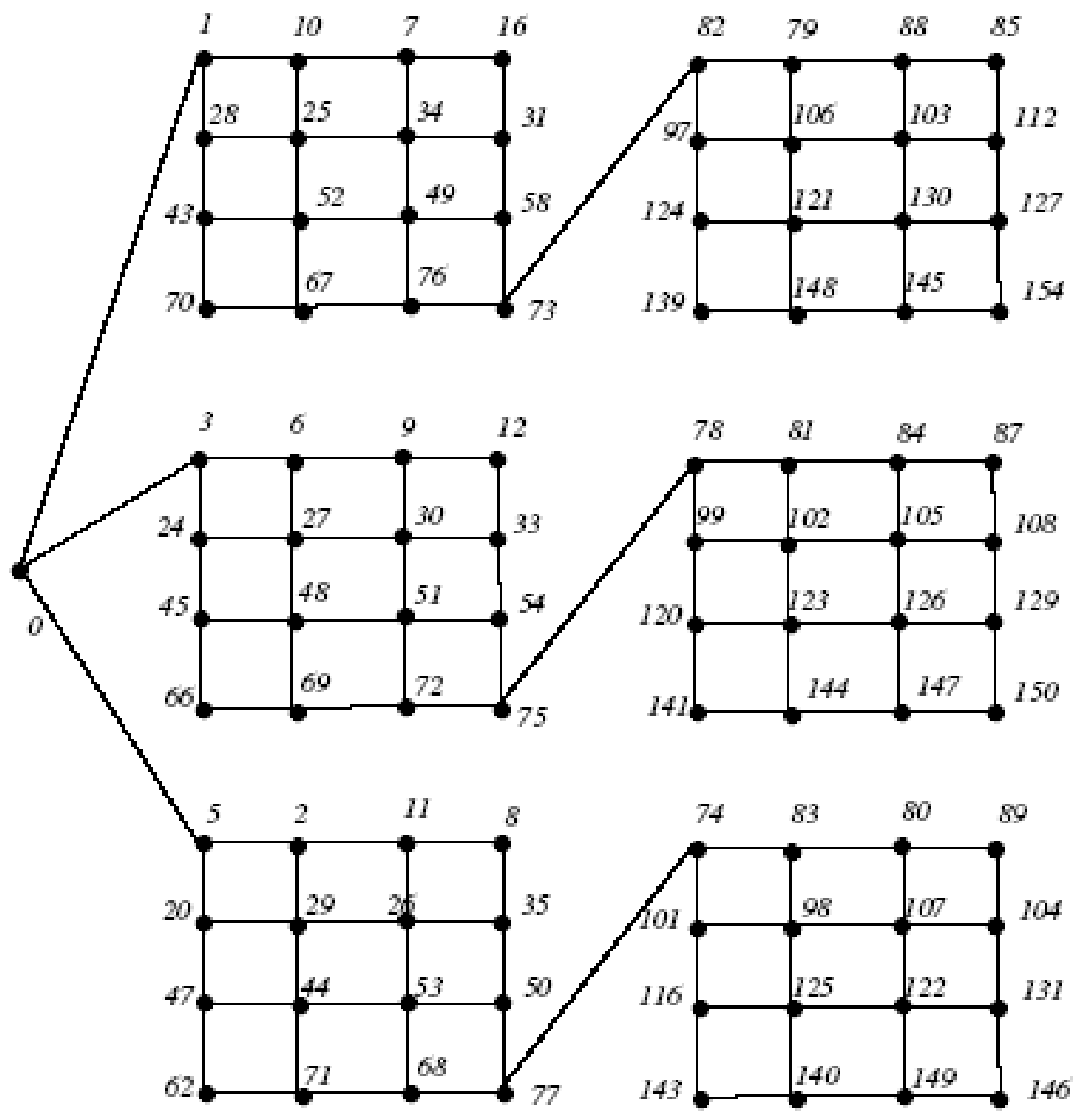

Figure 7:An odd harmonious labeling of $P_{2}^{3}\left(3.2 . P_{4} \times P_{4}\right)$. 


\section{Concluding Remarks}

In this work, we establish that path union of t copies of $P_{m} \times P_{n}$, path union of $\mathrm{t}$ different copies of $P_{m_{i}} \times P_{n_{i}}$ where $1 \leq i \leq t$, vertex union of $\mathrm{t}$ copies of $P_{m} \times P_{n}$, vertex union of $\mathrm{t}$ different copies of $P_{m_{i}} \times P_{n_{i}}$ where $1 \leq i \leq t$, one point union of path of $P_{n}^{t}\left(t . n . P_{m} \times P_{m}\right)$, t super subdivision of grid graph $P_{m} \times P_{n}$ admit an odd harmonious labeling. Further, we proceed with to prove more families of graphs are odd harmonious.

\section{Acknowledgement}

The authors thank the referee for the valuable comments to improve the presentation of the paper.

\section{References}

[1] J. Gallian, "A Dynamic Survey of Graph Labeling”, 20th ed. The Electronics Journal of Combinatorics, vol. \# DS6, p. 432, 2007. [On line]. Available: http://bit.ly/33bdu1B

[2] R. Graham and N. Sloane, "On Additive Bases and Harmonious Graphs", SIAM Journal on Algebraic Discrete Methods, vol. 1, no. 4, pp. 382-404, 1980, doi: 10.1137/0601045.

[3] F. Harary, Graph theory. Reading, MA: Addison-Wesley, 1972.

[4] Z. Liang and Z. Bai, "On the odd harmonious graphs with applications", Journal of Applied Mathematics and Computing, vol. 29, no. 1-2, pp. 105-116, Oct. 2008, doi: 10.1007/s12190-008-0101-0.

[5] P. Jeyanthi, S. Philo and K. Sugeng, "Odd harmonious labeling of some new families of graphs", SUT Journal of Mathematics, vol. 51, no. 2, pp. 53-65, 2015. [On lime]. Available: http://bit.ly/2T0pQoR

[6] P. Jeyanthi and S. Philo, "Odd harmonious labeling of some cycle related graphs”, Proyecciones (Antofagasta), vol. 35, no. 1, pp. 85-98, Mar. 2016, doi: 10.4067/S0716-09172016000100006.

[7] P. Jeyanthi and S. Philo, "Odd Harmonious Labeling of Plus Graphs", Bulletin of the International Mathematical Virtual Institute, vol. 7, no. 3, pp. 515-526, Apr. 2017. [On line]. Available: http://bit.ly/2TceXAt 
[8] P. Jeyanthi, S. Philo, and M. K. Siddiqui, "Odd harmonious labeling of super subdivisión graphs", Proyecciones (Antofagasta), vol. 38, no. 1, pp. 1-11, Feb. 2019, doi: 10.4067/S0716-09172019000100001.

[9] P. Jeyanthi and S. Philo, "Odd Harmonious Labeling of Some New Graphs", Southeast Asian Bulletin of Mathematics, vol. 43, no. 4, pp. 509-523, 2019. [On line] Available: http://bit.ly/31spYAw

[10] P. Jeyanthi and S. Philo, "Odd Harmonious Labeling of Subdivided Shell Graphs", International Journal of Computer Sciences and Engineering, vol. 7, (special issue), no. 5, pp. 77-80, Mar. 201, doi: 10.26438/ijcse/v7si 5.7780 .

[11] P. Jeyanthi and S. Philo, "Odd Harmonious Labeling of Certain Graphs", Journal of Applied Science and Computations, vol. 6, no.4, pp. 1224-1232, Apr. 2019. [On line]. Available: http://bit.ly/2Knk10k

[12] P. Jeyanthi and S. Philo, "Some Results on Odd Harmonious Labeling", Bulletin of the International Mathematical Virtual Institute, vol. 9, no. 3 pp. 567-576, May 2019. [On line]. Available: http://bit.ly/2T0sB9H

[13] P. Selvaraju, P. Balaganesan and J. Renuka, "Odd Harmonious Labeling of Some Path Related Graphs", International Journal of Math. Sci. \& Engg. Appls, vol. 7 no. 3, pp. 163-170, May 2013.

[14] S. Vaidya and N. Shah, "Some New Odd Harmonious Graphs", International Journal of Mathematics and Soft Computing, vol. 1, no. 1 pp. 9-16, 2011. [On line]. Available: http://bit.ly/2T6Us83

[15] S. Vaidya and N. Shah, "Odd Harmonious Labeling of Some Graphs", International Journal of Mathematical Combinatorics, vol. 3, pp. 105-112, Sep. 2012. [On line]. Available: http://bit.ly/2KkEcwo 